American Journal of Applied Sciences 6 (11): 1909-1914, 2009

ISSN 1546-9239

(C) 2009 Science Publications

\title{
Synthesis and Characterization of Silver/Clay Nanocomposites by Chemical Reduction Method
}

\author{
${ }^{1}$ Mansor Bin Ahmad, ${ }^{1}$ Kamyar Shameli, ${ }^{2}$ Majid Darroudi, \\ ${ }^{1}$ Wan Md Zin Wan Yunus and ${ }^{1}$ Nor Azowa Ibrahim \\ ${ }^{1}$ Department of Chemistry, Faculty of Science, University Putra Malaysia, \\ 43400 UPM Serdang, Selangor, Malaysia \\ ${ }^{2}$ Advanced Materials and Nanotechnology Laboratory, Institute of Advanced Technology, \\ University Putra Malaysia, 43400 UPM Serdang, Selangor, Malaysia
}

\begin{abstract}
Problem statement: Silver Nanoparticles (Ag-NPs) have been synthesized by using chemical reduction method into the interlayer space of a Montmorillonite (MMT) as a solid support which is used to antibacterial application and polymer nanocomposites for fabrication of medical devices. Approach: $\mathrm{AgNO}_{3}$ and $\mathrm{NaBH}_{4}$ were used as a silver precursor and reducing agent, respectively. The properties of Ag/MMT nanocomposites were studied as a function of the $\mathrm{AgNO}_{3}$ concentration. The crystalline structure, d-spacing of interlayer of MMT, the size distributions and surface plasmon resonance of synthesized Ag-NPs were characterized using Powder X-Ray Diffraction (PXRD), Transmission Electron Microscopy (TEM) and UV-vis spectroscopy. Results: The results obtained from UV-vis spectroscopy of synthesized Ag-NPs showed that the intensity of the maximum wavelength of the plasmon peaks were increased with the increasing in the $\mathrm{AgNO}_{3}$ concentration. The obtained information from UV-vis spectra of Ag-NPs was in an excellent agreement with the obtained microstructures studies performed by Transmission Electron Microscopy (TEM) and their size distributions. The prepared Ag/MMT nanocomposites are very stable over a long period of time in aqueous solution. Conclusion: The synthesized Ag/MMT nanocomposites are very stable in aqueous solution over a long period of time without any sign of precipitation. Silver nanoparticles in MMT suspension could be suitable to use antibacterial applications, since MMT is viewed as ecologically and environmentally inert material and used for biological application such as cosmetics and pharmaceutical usage.
\end{abstract}

Key words: nanocomposites, silver nanoparticles, montmorillonite, powder X-ray diffraction

\section{INTRODUCTION}

Nanoparticles are important materials for fundamental studies and diversified technical applications because of their size dependent properties or highly active performance due to the large surface areas, but when pure nanoparticles are used alone, they present some common problems, e.g., agglomeration between nanoparticles ${ }^{[1]}$. To overcome agglomeration, preparation of nanoparticles based on clay compounds, in which nanoparticles are supported within the interlamellar spaces of clay and/or on its external surfaces, is one of the most effective solutions ${ }^{[2-4]}$.

Recently, the preparations of metal nanoparticles were discussed in a great number of publications due to their unique physical and chemical properties which lead to many potential applications ${ }^{[5]}$. There are many preparation methods for the synthesis of metal nanoparticles such as the photo reduction or chemical reduction in aqueous medium with various polymer surfactants ${ }^{[6,7]}$, chemical reduction in soft matrixes e.g., reverse micelles ${ }^{[8]}$ or in solid matrices e.g., mesoporous silicate $^{[9]}$ and chemical vapor deposition ${ }^{[10]}$. Some of metal nanoparticles have also been synthesized in the two-dimensional interlayer space of Montmorillonite $(\mathrm{MMT})^{[11-13]}$. MMT as lamellar clay has intercalation, swelling and ion exchange properties. Its interlayer space has been used for the synthesis of nanoparticles materials and biomaterials, as support for anchoring transition-metal complex catalysts and as adsorbents for cationic ions ${ }^{[14]}$. Silver Nanoparticles (Ag-NPs) are widely used as photosensitive components ${ }^{[15]}$, catalysts $^{[16-20]}$, photo-catalysts ${ }^{[21-24]}$ and in surface enhanced Raman spectroscopy ${ }^{[25-27]}$ as well as in

Corresponding Author: Mansor Bin Ahmad, Department of Chemistry, Faculty of Science, University Putra Malaysia, 43400 UPM Serdang, Selangor, Malaysia Tel.: (603)89466044 Fax: (603)89466043 
chemical analysis. In this research, we have synthesized Ag-NPs into interlayer of MMT in aqueous solutions by using $\mathrm{NaBH}_{4}$ as a reducing agent at room temperature.

\section{MATERIALS AND METHODS}

Materials: All reagents were of analytical grades and were used as received without further purification. $\mathrm{AgNO}_{3}$ (99.98\%), used as silver precursor, was supplied from Merck, Germany. MMT, used as a solid support for Ag-NPs, was purchased from Kunipa-F, Japan. $\mathrm{NaBH}_{4}(98.5 \%$, Sigma-Aldrich, USA) was used as a reducing agent. All aqueous solutions were prepared with double distilled water (DD-water).

Synthesis of Ag/MMT nanocomposites: For the synthesis of $\mathrm{Ag} / \mathrm{MMT}$ nanocomposites, the silver contents of the samples were 0.5 (S1), 1.0 (S2), 1.5 (S3), 2.0 (S4) and $5.0 \mathrm{~g}$ (S5) Ag/100 g MMT. Constant amounts of MMT were suspended in different volumes of $1 \times 10^{-3} \mathrm{M} \mathrm{AgNO}_{3}$ solution and stirred for $24 \mathrm{~h}$. Freshly prepared $\mathrm{NaBH}_{4}\left(4 \times 10^{-2} \mathrm{M}\right)$ solution was then added to the suspensions under continuous stirring to reach a constant $\mathrm{AgNO}_{3} / \mathrm{NaBH}_{4}$ molar ratio (1:4). After the addition of the reducing agent stirring was continued for another one hour. The suspensions were finally centrifuged, washed with DD-water twice and dried under vacuum overnight. All experiments were conducted at ambient temperature.

Characterization methods and instrumentation: The prepared Ag/MMT nanocomposites were characterized by using Ultraviolet-Visible (UV-vis) spectroscopy, Transmission Electron Microscopy (TEM) and Powder X-Ray Diffraction (PXRD). The UV-vis spectra were recorded over the range of 300$700 \mathrm{~nm}$ by a Lambda 25-Perkin Elmer UV-vis spectrophotometer. TEM observations were carried out on a Hitachi H-7100 electron microscopy and the particle size distributions were determined using the UTHSCSA Image Tool software, Version 3.00 program. The structures of produced Ag/MMT nanocomposites were carried out on a Philips PXRD (X'pert, $\mathrm{Cu} \mathrm{K} \mathrm{K}_{\alpha}$ radiation). The change in interlamellar spacing of MMT and Ag/MMT nanocomposite were also studied by using PXRD in the small angle range of $2^{\circ}<2 \theta<12^{\circ}$. The interlamellar space was calculated from the PXRD peak positions using Bragg's equation. A wavelength equal to $0.15418 \mathrm{~nm}$ was used for these measurements. The PXRD patterns were recorded at a scan speed of $2^{\circ} \min ^{-1}$. After reactions the samples were centrifuged by using high speed centrifuge machine (Avanti J25, Beckman).

\section{RESULTS}

The formation of Ag-NPs was followed by measuring the surface plasmon resonance (SPR) of the MMT suspensions containing Ag-NPs at the wavelength ranged from 300-700 nm (Fig. 1). TEM images and their size distributions of Ag-NPs show the mean diameter of the nanoparticles ranged from about 4-9 nm (Fig. 2). The comparison between PXRD patterns of MMT and prepared Ag/MMT nanocomposite under chemical reduction route in the small angle range of $2 \theta\left(2^{\circ}<2 \theta<12^{\circ}\right)$ indicate the formation of the intercalated structure (Fig. 3). The PXRD patterns were also employed to determine the crystalline structures of the synthesized Ag-NPs (Fig. 4). The stability of synthesized MMT suspensions containing Ag-NPs was found to be stable over a long period of time (>2 months) without any sign of precipitation.

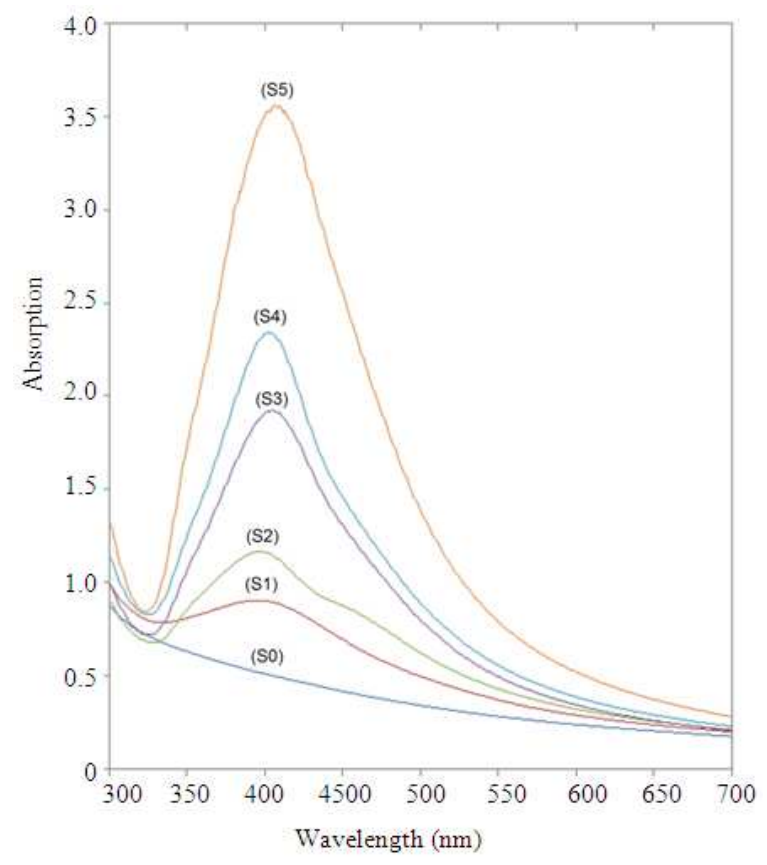

Fig. 1: UV-vis absorption spectra of Ag/MMT suspension for different $\mathrm{AgNO}_{3}$ concentrations; (S1) $0.5 \%$, (S2) $1.0 \%$, (S3) $1.5 \%$, (S4) $2.0 \%$, (S5) $5.0 \%$ and (S0) $\mathrm{AgNO}_{3} / \mathrm{MMT}$ suspension in the absence of $\mathrm{NaBH}_{4}$ 


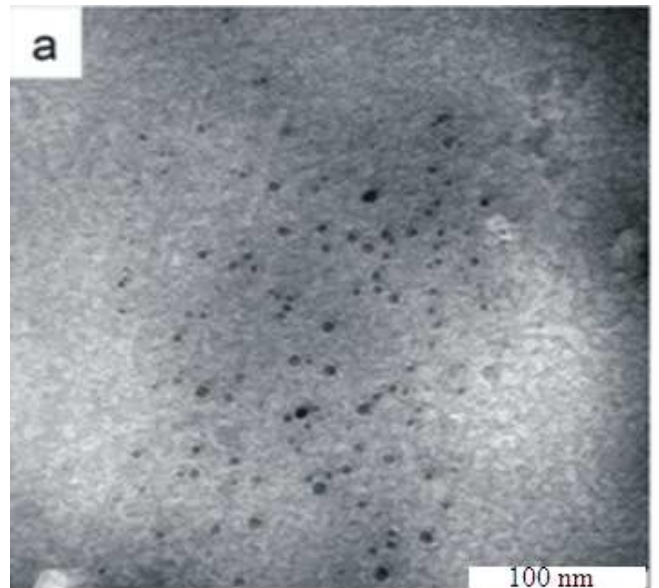

(a)

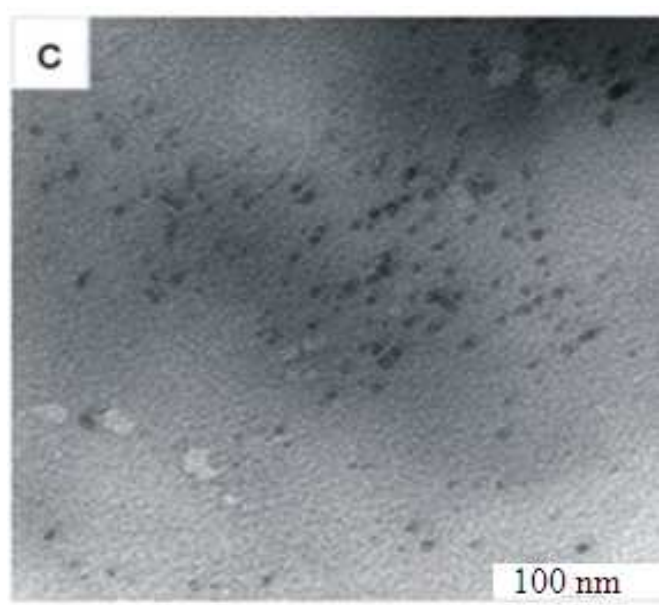

(c)

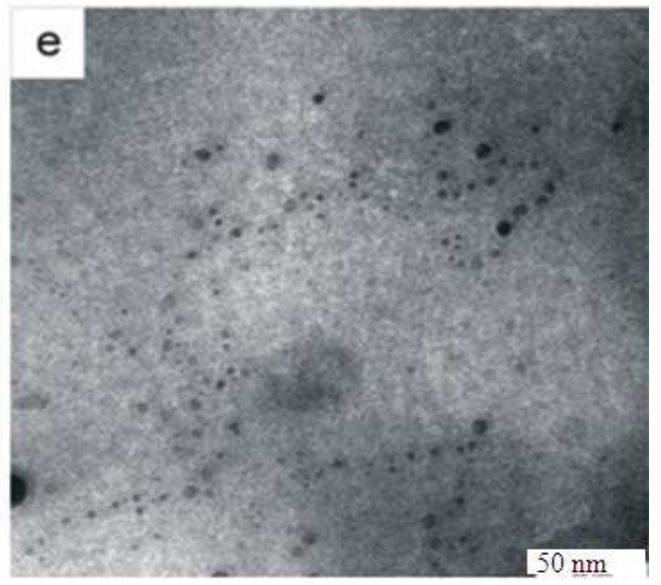

(e)

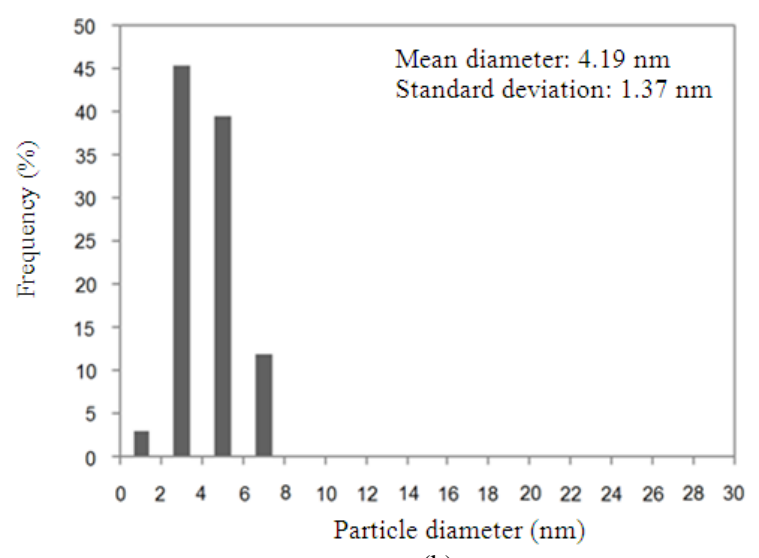

(b)

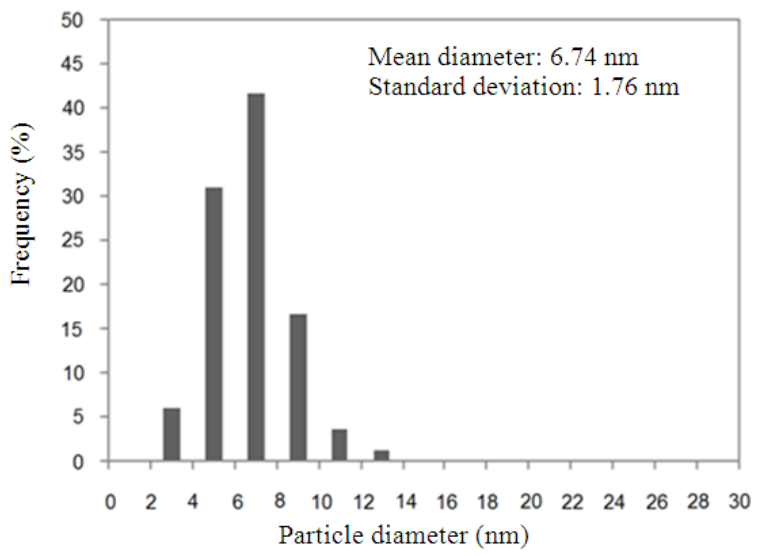

(d)

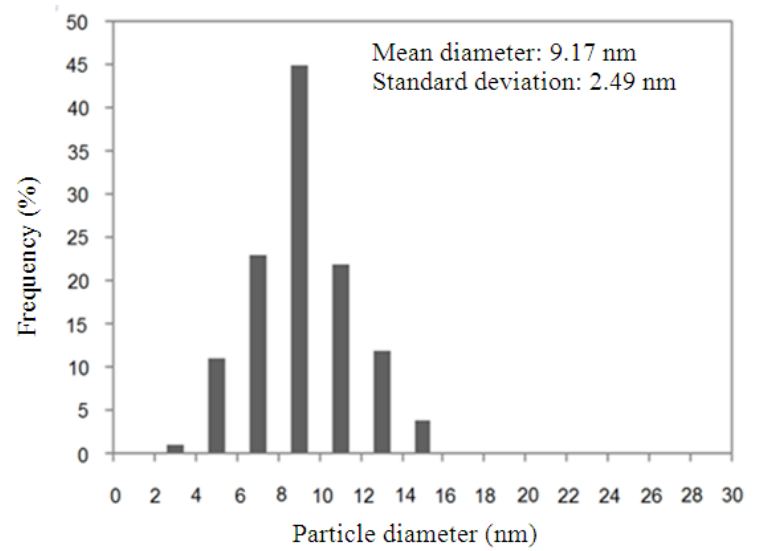

(f)

Fig. 2: TEM images and their corresponding particle size distributions of $\mathrm{Ag} / \mathrm{MMT}$ nanocomposites at different $\mathrm{AgNO}_{3}$ concentrations [1.0\% (a-b), 2.0\% (c-d) and 5.0\% (e-f)] 
Am. J. Applied Sci., 6 (11): 1909-1914, 2009

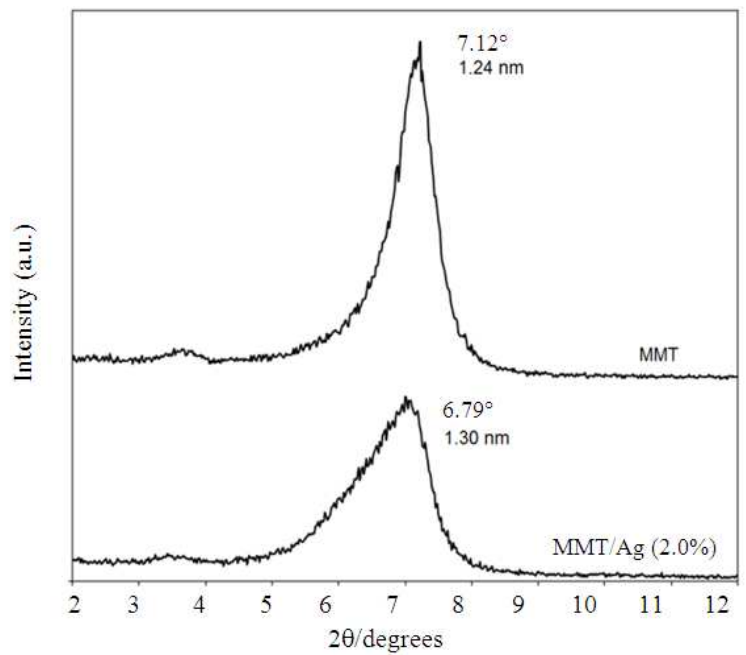

Fig. 3: PXRD patterns of MMT and Ag/MMT nanocomposite for determination of $\mathrm{d}$-spacing $\left(\mathrm{d}_{\mathrm{L}}\right)$

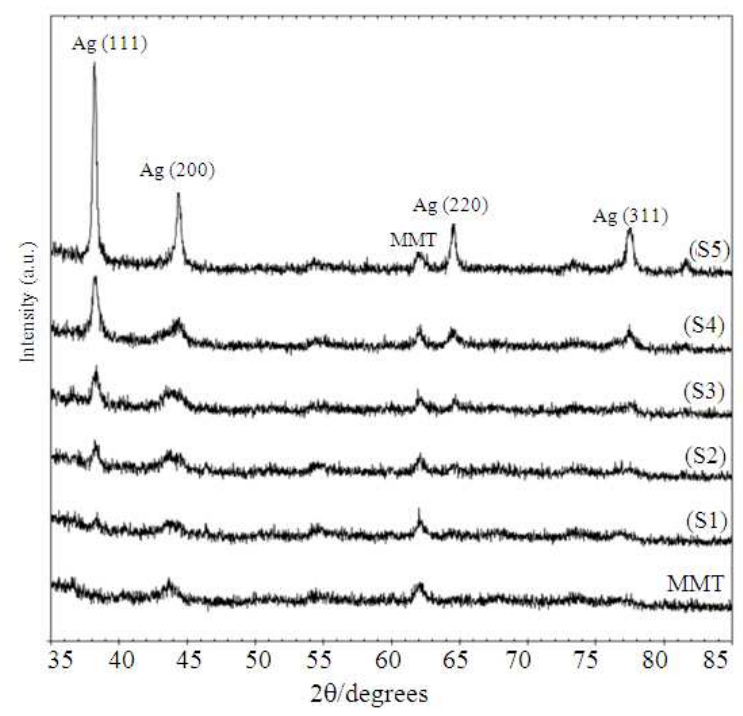

Fig. 4: PXRD patterns of MMT and Ag-NPs into MMT as a substrate at different $\mathrm{AgNO}_{3}$ concentrations

\section{DISCUSSION}

The color of $\mathrm{AgNO}_{3} / \mathrm{MMT}$ suspensions through reduction process by $\mathrm{NaBH}_{4}$ changed from colorless to light brown, then to brown and finally to green, indicating the formation Ag-NPs in MMT suspension.

The characteristic silver SPR bands were detected around $400 \mathrm{~nm}$ (Fig. 1). These absorption bands were assumed to correspond to the Ag-NPs smaller than
$10 \mathrm{~nm}^{[6,7]}$. While there is no characteristic UV-vis absorption of Ag-NPs before addition of $\mathrm{NaBH}_{4}$ (Fig. 1f), growth of the plasmon peak at $396 \mathrm{~nm}$ indicates the formation of Ag-NPs in S1 (Fig. 1a). Gradual increase in $\mathrm{AgNO}_{3}$ concentration, from S1-S4, increases the corresponding peak intensities (Fig. 1a-d) in a range of wavelengths from 396-402 $\mathrm{nm}$. In S5, the absorption peak due to SPR of Ag was slightly redshifted to higher wavelength $(404 \mathrm{~nm})$ indicating the increase in the size of $\mathrm{Ag}-\mathrm{NPs}^{[28]}$. TEM images and their size distributions of Ag-NPs also confirmed this phenomenon (Fig. 2).

As shown in Fig. 3 the original d-spacing $\left(d_{L}\right)$ of MMT, $1.24 \mathrm{~nm}$ is increased to $1.30 \mathrm{~nm}$ at smaller $2 \theta$ angles $\left(2 \theta=6.79^{\circ}\right.$ for S4) by silver intercalation. This $d_{L}$ value is a direct proof of the fact that, in the path of ion exchange, $\mathrm{Ag}^{+}$ions are bound not only on the external surfaces and edges of MMT but also in the interlamellar space. Metallic nanoparticles formed at the latter location are to cause of the increase in basal spacing. In these samples the intensities of the reflections are significantly lower whereas their halfwidths are larger than those of undoped clay minerals: the highly ordered parallel lamellar structure of the mineral is disrupted by particle formation ${ }^{[29]}$.

All the nanocomposites have similar diffraction profiles and the PXRD peaks at $2 \theta$ of $38.28^{\circ}$, $44.38^{\circ}, 64.52^{\circ}$ and $77.61^{\circ}$ (Fig. 4) can be attributed to the (111), (200), (220) and (311) crystallographic planes of face-centered cubic (fcc) silver crystals, respectively ${ }^{[30]}$. For all samples, the main crystalline phase was silver and no obvious other phases as impurities were found in the PXRD patterns. The PXRD peak broadenings of Ag-NPs are mostly because of existing of nano-sized particles in these nanocomposites ${ }^{[31]}$. In addition there is a characteristic peak in about $2 \theta=62.1^{\circ}$ that related to MMT (PXRD Ref. \#00-003-0010) as a stable substrate.

\section{CONCLUSION}

Synthesis of Ag-NPs in the interlamellar space of MMT in wet chemical reduction method by using $\mathrm{NaBH}_{4}$ as a reducing agent at room temperature is simply possible. The properties of Ag-NPs were studies as a function of $\mathrm{AgNO}_{3}$ concentration which the molar ratio of $\mathrm{AgNO}_{3} / \mathrm{NaBH}_{4}$ was constant. When the amount of $\mathrm{AgNO}_{3}$ had been increased, the particles size of AgNPs was gradually increased but their size distribution was narrow. The synthesized Ag/MMT nanocomposites are very stable in aqueous solution over a long period of time without any sign of precipitation. 


\section{REFERENCES}

1. Zhu, H.Y., J.A. Orthman, J.Y. Li, J.C. Zhao, G.J. Churchman and E.F. Vansant, 2002. Novel composites of $\mathrm{TiO}_{2}$ (anatase) and silicate nanoparticles. Chem. Mater., 14: 5037-5044. DOI: $10.1021 / \mathrm{cm} 0205884$

2. Choy, J.H., J.H. Park and J.B. Yoon, 1998. Multilayered $\mathrm{SiO}_{2} / \mathrm{TiO}_{2}$ nanosol particles in twodimensional aluminosilicate catalyst-support. J. Phys. Chem. B., 102: 5991-5995. DOI: 10.1021/jp9815863

3. Mogyorosi, K., I. Dékány and J.H. Fendler, 2003. Preparation and characterization of clay mineral intercalated titanium dioxide nanoparticles. Langmuir, 19: 2938-2946. DOI: 10.1021/la025969a

4. Miao, S. et al., 2006. Synthesis and characterization of $\mathrm{TiO}_{2}$-montmorillonite nanocomposites and their application for removal of methylene blue. J. Mater. Chem., 16: 579-584. DOI: 10.1039/b511426h

5. Papp, S., R. Patakfalvi and I. Dékány, 2008. Metal nanoparticle formation on layer silicate lamellae. Colloid Polymer Sci., 286: 3-14. DOI: 10.1007/s00396-007-1728-3

6. Aihara, N., K. Torigoe and K. Esumi, 1998. Preparation and characterization of gold and silver nanoparticles in layered laponite suspensions: Langmuir, $\quad 14$ : 4945-4949. DOI: 10.1021/la980370p

7. Lin, X.Z., X. Teng and H. Yang, 2003. Direct synthesis of narrowly dispersed silver nanoparticles using a single-source precursor. Langmuir, 19: 10081-10085. DOI: $10.1021 / \mathrm{la} 035185 \mathrm{c}$

8. Taleb, A., C. Petit and M.P. Pileni, 1997. Synthesis of highly monodisperse silver nanoparticles from AOT reverse micelles: A way to $2 \mathrm{D}$ and $3 \mathrm{D}$ selforganization. Chem. Mater., 9: 950-959. DOI: $10.1021 / \mathrm{cm} 960513 \mathrm{y}$

9. Dag, Ö., O. Samarskays, N. Coombs and G.A. Ozin, 2003. The synthesis of mesostructured silica films and monoliths functionalised by noble metal nanoparticles. J. Mater. Chem., 13: 328-334. DOI: $10.1039 / b 209153 b$

10. Szlyk, E., P. Piszczek, A. Grodzicki, M. Chaberski, A. Golinski, J. Szatkowski et al., 2001. CVD of Ag-I complexes with tertiary phosphines and perfluorinated carboxylates-A new class of silver precursors. Chem. Vapor Deposit., 7: 111-116. DOI: $\quad 10.1002 / 1521-3862(200105) 7: 3<111:: A I D-$ CVDE111>3.0.CO;2-V
11. Kyotani, T., N. Sonobe and A.Tomita, 1988. Formation of highly orientated graphite from polyacrylonitrile by using a two-dimensional space between montmorillonite lamellae: Nature, 331: 331-333. DOI: $10.1038 / 331331 \mathrm{a} 0$

12. Bakandritsos, A., T. Steriotis and D. Petridis, 2004. High surface area montmorillonite-carbon composites and derived carbons. Chem. Mater., 16: 1551-1559. DOI: $10.1021 / \mathrm{cm} 0350030$

13. Darroudi, M., M.B. Ahmad, K. Shameli, A.H. Abdullah and N.A. Ibrahim, 2009. Synthesis and characterization of UV-irradiated silver/montmorillonite nanocomposites. Solid State Sci., 11: 1621-1624. DOI: 10.1016/j.solidstatesciences.2009.06.016

14. Kozak, M. and L.J. Domka, 2004. Adsorption of the quaternary ammonium salts on montmorillonite. J. Phys. Chem. Solids, 65: 441-445. DOI: 10.1016/j.jpcs.2003.09.015

15. Hailstone, R.K., 1995. Computer simulation studies of silver cluster formation on $\mathrm{AgBr}$ microcrystals. J. Phys. Chem., 99: 4414-4428. DOI: $10.1021 / \mathrm{j} 100013 \mathrm{a} 009$

16. Sun, T. and K. Seff, 1994. Silver clusters and chemistry in zeolites. Chem. Rev., 94: 857-870. DOI: $10.1021 / \mathrm{cr} 00028 \mathrm{a} 001$

17. Verykios, X.E., F.P. Stein and R.W. Coughlin, 1980. Oxidation of ethylene over silver: Adsorption, kinetics, catalyst. Catal. Rev. Sci. Eng., $\quad 22$ : 197-234. DOI: $10.1080 / 03602458008066534$

18. Claus, P. and H. Hofmeister, 1999. Electron microscopy and catalytic study of silver catalysts: structure sensitivity of the hydrogenation of crotonaldehyde. J. Phys. Chem. B., 103: 2766-2775. DOI: $10.1021 /$ jp983857f

19. Haneda, M., Y. Kintaichi, M. Inaba and H. Hamada, 1998. Infrared study of catalytic reduction of nitrogen monoxide by propene over $\mathrm{Ag} / \mathrm{TiO}_{2} \mathrm{ZrO}_{2}$. Catal. Today, 42: 127-135. DOI: 10.1016/S09205861(98)00083-2

20. Goncharova, S.N., E.A. Paukshtisand B.S. Balzhinimaev, 1995. Size effects in ethylene oxidation on silver catalysts. Influence of support and Cs promoter. Applied Catal. A: General, 126: 67-84. DOI: 10.1016/0926-860X(95)00036-4

21. Sclafani, A., M. Mozzanega and P. Pichat, 1991. Effect of silver deposits on the photocatalytic activity of titanium dioxide samples for the dehydrogenation or oxidation of 2-propanol. J. Photochem. Photobiol. A: Chem., 59: 181-189. DOI: 10.1016/1010-6030(91)87006-H 
22. Tada, H., K. Teranishi, Y. Inubushi and S. Ito, 2000. Ag nanocluster loading effect on $\mathrm{TiO}_{2}$ photocatalytic reduction of $\mathrm{Bis}(2-$ dipyridyl)disulfide to 2-Mercaptopyridine by $\mathrm{H}_{2} \mathrm{O}$. Langmuir, 16 : 3304-3309. DOI: 10.1021/la991315z

23. Tada, H., K. Teranishi and S. Ito, 1999. Additive effect of sacrificial electron donors on $\mathrm{Ag} / \mathrm{TiO}_{2}$ photocatalytic reduction of $\mathrm{Bis}$ (2-dipyridyl) disulfide to 2-Mercaptopyridine in aqueous media. Langmuir, $\quad$ 15: 7084-7087. DOI: 10.1021/la981728k

24. Sclafani, A. and J. Herrmann, 1998. Influence of metallic silver and of platinum-silver bimetallic deposits on the photocatalytic activity of titania (anatase and rutile) in organic and aqueous media. J. Photochem. Photobiol. A: Chem., 113: 181-188. DOI: 10.1016/S1010-6030(97)00319-5

25. Shirtcliffe, N., U. Nickel and S. Schneider, 1999. Reproducible preparation of silver sols with small particle size using borohydride reduction: For use as nuclei for preparation of larger particles. J. Colloid Interface Sci., 211: 122-129. DOI:10.1006/jcis.1998.5980

26. Nickel, U., A.Z. Castell, K. Poppl and S. Schneider, 2000. A silver colloid produced by reduction with hydrazine as support for highly sensitive surface-enhanced raman spectroscopy. Langmuir, 16: 9087-9091. DOI: 10.1021/la000536y

27. Bright, R.M., M.D. Musick and M.J. Natan, 1998. Preparation and characterization of $\mathrm{Ag}$ colloid monolayers. Langmuir, 14: 5695-5701. DOI: 10.1021/la980138j
28. Liu, F.K., Y.C. Hsu, M.H. Tsai and T.C. Chu, 2007. Using $\gamma$-irradiation to synthesize Ag nanoparticles. Mater. Lett., 61: 2402-2405. DOI: 10.1016/j.matlet.2006.07.193

29. Patakfalvi, R., A. Oszko and I. Dékány, 2003. Synthesis and characterization of silver nanoparticle/kaolinite composites: Colloids and surfaces A. Physicochem. Eng. Aspects, 220: 45-54. DOI: 10.1016/S0927-7757(03)00056-6

30. Temgire, M.K. and S.S. Joshi, 2004. Optical and structural studies of silver nanoparticles. Radiat. Phys. Chem., 71: 1039-1044. DOI: 10.1016/j.radphyschem.2003.10.016

31. Prasad, V., C.D. Souza, D. Yadav, A.J. Shaikh and N. Vigneshwaran, 2006. Spectroscopic characterization of zinc oxide nanorods synthesized by solid-state reaction. Spectrochimica Acta Part A. Mol. Biomol. Spectrosc., 65: 173-178. DOI: 10.1016/j.saa.2005.10.001 